\title{
INFLUÊNCIA DE VARIÁVEIS AMBIENTAIS NA COMUNIDADE ARBÓREA DE INSELBERGS
}

\author{
Gabriela Gomes Pires ${ }^{1 *}$, Rubens Manoel dos Santos ${ }^{1}$, Rosângela Alves Tristão ${ }^{1}$, Daniel Salgado Pifano ${ }^{2}$, Carlos
} Alberto Reis ${ }^{1}$, Daniel Quedes Domingos ${ }^{1}$

*Autor para correspondência: gabyfloresta@gmail.com

RESUMO: Os inselbergs abrigam uma série de microclimas responsáveis pela diferenciação das condições ecológicas na paisagem, formando um complexo sistema de gradientes, onde muitas vezes pode ser caracterizada como uma área de ecótono. O objetivo desse estudo foi de caracterizar a estrutura da vegetação e correlaciona-la com as variáveis edáficas de diferentes ambientes, em um fragmento de Floresta Estacional Semidecidual Montana, no município de Coqueiral, MG. Foram lançadas 20 parcelas (40x10m e $20 \times 20 \mathrm{~m}$ ) e amostrados todos os indivíduos com $\mathrm{CAP} \geq 15,7 \mathrm{~cm}$. Para o estudo de estrutura foram avaliados dominância absoluta (DoA), densidade absoluta (DA), valor de cobertura (VC) e freqüência absoluta (FA). Além de uma análise de correspondência canônica (CCA) correlacionando os valores de abundância com variáveis do solo. Encontrou-se uma relação entre o gradiente de distribuição das espécies com as características do solo. A mata ciliar apresentou maior diferença na abundância de espécies em relação às características do solo.

Palavras-chave: CCA, gradiente ambiental, estrutura de comunidade.

\section{INFLUENCE OF ENVIRONMENTAL VARIABLES IN THE COMMUNITY OF ARBOREAL INSELBERGS}

\begin{abstract}
The inselbergs shelter a series of microclimates responsible for the differentiation of ecological conditions in the landscape, forming a complex system of gradients, which can often be characterized as an area of ecotone. The aim of this study was to characterize the vegetation structure and correlates it with the soil characteristics of different environments, in a fragment of semideciduous forest Montana, in the city of Coqueiral, MG. Have been launched 20 plots (40x10m and 20x20m) and sampled all individuals with $C A P \geq 15.7 \mathrm{~cm}$. For the study of structure have been evaluated absolute dominance (DoA), absolute density (AD), cover $(V C)$ and absolute frequency $(A F)$. In a canonical correspondence analysis (CCA) correlating the values of abundance with soil variables. There was a relationship between the gradient distribution of species with soil characteristics, riparian vegetation showed the greatest difference in abundance species in relation to the soil characteristics.
\end{abstract}

Key words: CCA, environmental gradient, community structure

\section{INTRODUÇÃO}

Ilhas de afloramentos rochosos, inselbergs, ocorrem ao longo de todas as principais zonas climáticas e vegetacionais, mas são particularmente abundantes em algumas regiões tropicais. Apesar de algumas vezes possuírem uma ocorrência ampla e dominante ao longo de algumas paisagens, a história da sua exploração biológica através de estudos é relativamente curta (POREMBSKI, 2007), sobretudo aos que se referem a levantamentos arbóreos em inselbergs.

Estas ilhas abrigam uma série de microclimas responsáveis pela diferenciação das condições ecológicas nesses ambientes, gerando assim microhabitats contrastando com a paisagem ("Matriz") (POREMBSKI, 2000;
PARMENTIER; HARDY, 2009; PARMENTIER, 2005). A "matriz" corresponde a toda vegetação que se encontra próxima aos inselbergs, e as semelhanças florísticas entre esses ambientes são importantes fontes de propágulos e de regeneração ou funciona como sumidouros, conceitos importantes na ecologia da restauração (JOLY, 1970; BURKE, 2002; GOMES; ALVES, 2010).

As variações microclimáticas em inselbergs são ocasionadas principalmente pelas condições de altas temperaturas, baixa umidade, maior exposição a ventos, retenção de calor e maior escoamento de água devido a sua inclinação íngreme (POREMBSKI, 2007). As condições microclimáticas juntamente com a baixa disponibilidade de nutrientes tornam-se os principais responsáveis pelos processos ecológicos em inselbergs, capazes de

${ }^{1}$ Universidade Federal de Lavras - Lavras, Minas Gerais, Brasil

${ }^{2}$ Universidade Federal do Vale do São Francisco - Petrolina, Pernambuco, Brasil

Cerne, Lavras, v. 20, n. 1, p. 97-104, jan./mar. 2014 
determinarem a estrutura e composição da vegetação nessas ilhas de afloramentos rochosos (PARMENTIER, 2005; GOMES; ALVES, 2010; POREMBSKI, 2007; GROGER; HUBER, 2007).

Sob uma perspectiva local, os inselbergs apresentam uma composição de espécies de plantas que é melhor explicada por sua correlação com as condições edáficas ao longo do ambiente. Os inselbergs podem ser caracterizados como um ecótono, gerando uma grande variedade de tipos de habitats. Já em escala regional, devido a fragmentação, influenciam na distribuição espacial das comunidades e na dispersão dos indivíduos. Sendo importantes para determinar a composição florística destes locais (PARMENTIER et al., 2005).

O presente estudo teve como objetivo diagnosticar a composição de espécies arbóreas em um inselberg relacionado com variáveis edáficas e com a vegetação circunvizinha, mata ciliar e corredor de vegetação, em uma área localizada em Coqueiral, sul de Minas Gerais.

\section{MATERIAL E MÉTODOS}

\section{1 Área de estudo}

O estudo foi conduzido em 2006. O inventário estrutural foi realizado no município de Coqueiral- $\mathrm{MG}$, situado a $21^{\circ} 09^{\prime} 19^{\prime}$ ' S e $45^{\circ} 28^{\prime} 17^{\prime \prime} \mathrm{W}$, com uma altitude variando de 810 a $840 \mathrm{~m}$ (Figura 1). O clima caracterizase por ser do tipo mesotérmico, tendo uma média anual de precipitação de $1.493 \mathrm{~mm}$ e de temperatura $19,3{ }^{\circ} \mathrm{C}$ (VILELA; RAMALHO, 1979). Aárea insere-se no domínio da Mata Atlântica e constitui-se de Floresta Estacional Semidecidual Montana (OLIVEIRA-FILHO; FONTES, 2000). O fragmento florestal amostrado apresenta um mosaico de ambientes e vegetação. É composto por áreas de afloramentos rochosos de granito-gnaisse, denominado inselbergs; mata ciliar e corredor de vegetação localizada nas linhas de drenagem, ligando os inselbergs à mata ciliar.

As parcelas foram lançadas com o intuito de captar as maiores variações ambientais, tendo sua distribuição: 1-6, parcelas de 10x40 m localizadas na mata ciliar; 7-15, parcelas de 10x40 m em corredor de vegetação; 15-20, parcelas de $20 \times 20 \mathrm{~m}$ situadas em inselbergs, perfazendo uma área de 0,8 ha. Nas parcelas foram mensurados e identificados todos os indivíduos com CAP (circunferência a altura do peito a $1,30 \mathrm{~m}$ ) maior que $15,7 \mathrm{~cm}$. No centro geométrico de cada parcela foram coletadas amostras compostas $(0-20 \mathrm{~cm})$ de solo, as quais foram feitas análises

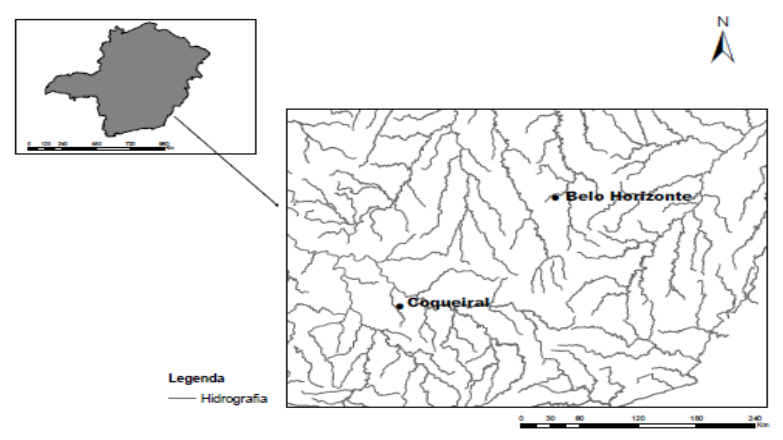

Figura 1- Mapa de localização da área de estudo em Coqueiral, MG.

Figure 1- Location map of study area in Coqueiral MG.

químicas obtendo as variáveis: $\mathrm{pH}$ em água; teores de Potássio (K), Fósforo (P), Cálcio (Ca), Magnésio (Mg) e Alumínio (Al); Acidez Potencial $(\mathrm{H}+\mathrm{Al})$, Soma de Bases (SB), CTC efetiva (t), CTC a pH 7,0 (T), saturação por Alumínio (m), Saturação por Bases (V), Carbono (C), Matéria Orgânica (MO), e proporções de Areia, Silte e Argila, realizadas no Laboratório de Análise de Solos da Universidade Federal de Lavras, seguindo os procedimentos do protocolo da EMBRAPA (2002).

\subsection{Análise de dados}

Para descrever a estrutura da floresta, foram calculados os seguintes parâmetros estruturais: densidade absoluta (DA), frequência absoluta (FA), dominância absoluta (DoA) e valor de cobertura (VC) (MUELLERDOMBOIS; ELLEMBERG, 1974). Para analisar a diversidade foi calculado o índice de Shannon e equabilidade de Pielou. E para detectar possíveis diferenças entre os indivíduos arbóreos em cada ambiente foi contabilizado também, a porcentagem de indivíduos perfilhados em cada ambiente.

A similaridade entre as parcelas foi calculada a partir dos valores de abundância através do método de ligação simples usando o coeficiente de Bray-Curtis. $\mathrm{O}$ resultado foi exibido por meio de um dendrograma, processado pelo programa PAST 1.94 (HAMMER, 2009).

Ao analisar as correlações entre a abundância das espécies arbóreas e as variáveis ambientais, referente ao solo, foi feita uma análise de correspondência canônica (CCA) utilizando uma matriz de abundância de espécies. Esta análise multivariada é atualmente a mais indicada quando o objetivo é obter uma relação mais

Cerne, Lavras, v. 20, n. 1, p. 97-104, jan./mar. 2014 
estreita das variáveis ambientais com a abundância de espécies (FELFILI et al., 2007). Após realizar uma CCA preliminar foram eliminadas as variáveis que apresentaram redundância entre si. Restaram, na CCA final, cinco variáveis ambientais: $\mathrm{pH}$, Cálcio $(\mathrm{Ca})$, Alumínio $(\mathrm{Al})$, soma de bases (SB), matéria orgânica (MO).

Juntamente com a CCA, foi realizada a análise de Monte Carlo que consiste em permutar aleatoriamente as linhas da matriz de variáveis ambientais com o intuito de testar a significância da correlação entre as duas matrizes, identificando a probabilidade de acerto da relação encontrada entre as matrizes originais (SOUZA et al., 2003). Para caracterizar melhor cada ambiente foi feita uma análise de espécie indicadora, afim de detectar quais espécies melhor caracterizam cada ambiente. Para isso foi feito uma matriz de abundância de espécies, onde as espécies que apresentaram valores significativos para o teste de Monte Carlo, p menor ou igual a 0,05, foram selecionadas. As análises de espécies indicadoras e CCA foram realizadas pelo programa PC-ORD 5 for Windows.

\section{RESULTADOS E DISCUSSÃO}

No total foram amostrados 1264 indivíduos, pertencentes a 160 espécies e 53 famílias botânicas. A família com maior representatividade em todo levantamento foi Fabaceae com 17,33\%, seguida de Myrtaceae e Rubiaceae com $16,14 \%$ e $12,58 \%$, respectivamente. Do total das espécies amostradas 83 foram encontradas na mata ciliar pertencentes a 35 famílias sendo Fabaceae a mais abundante. No corredor de vegetação 101 espécies e 34 famílias foram catalogadas, onde Myrtaceae foi a família com maior representatividade. Nos inselberg, Myrtaceae correspondeu a família mais abundante das 64 espécies (Tabela 1).

Os parâmetros fitossociológicos, dominância absoluta (DoA) e densidade absoluta (DA) são ferramentas utilizadas para demonstrar como a vegetação se comporta horizontalmente dentro da comunidade. Nos inselbergs encontraram-se a maior quantidade de indivíduos arbóreos, $\mathrm{DA}=1890.00$ ind.ha-1, DoA=21.58 m2.ha-1. A mata ciliar e o corredor de vegetação apresentaram, DA=1191.67 ind. ha- 1 ; DoA= $28.58 \mathrm{~m} 2$.ha-1 ; $\mathrm{DA}=1666.67$ ind.ha-1 ; $\mathrm{DoA}=$ $29.57 \mathrm{~m} 2$.ha-1 respectivamente. Os menores diâmetros dos indivíduos amostrados no inselberg podem estar associados às condições de escassez de água e baixa disponibilidade de nutrientes (POREMBSKI, 2007).

Analisando a estrutura e composições do levantamento arbóreo, Copaifera langsdorffii apresentou maior DA, assim como FR. No entanto a espécie Callisthene major apresentou maior área basal. Com isso podemos concluir que C. langsdorffii possui maior ocorrência na área, mas não apresenta a maior ocupação em ralação a área. Pinheiro e Monteiro (2009), caracterizaram esta espécie como ambígua, sendo encontrada em distintos ambientes como cerrado e interior de mata. Padrão semelhante foi descrito por Oliveira-Filho e Ratter (2000), podendo ser encontrado em formações florestais, ciliares e savânicas (LOBO; JOLY, 2000).

Em cada ambiente as espécies apresentaram diferentes parâmetros fitossociológicos. Na área de mata ciliar, Croton floribundus apresentou maior DA. A composição florística em matas ciliares possui características peculiares sendo estas adaptadas ou tolerantes a solos encharcados ou inundados. Analisando área basal e frequência relativa nas parcelas de mata ciliar, Platycyamus regnellii e Metrodorea stipularis apresentaram os maiores valores. Nas parcelas correspondentes a área de inselberg, Copaifera langsdorffi apresentou maior DA, Zanthoxylum rhoifolium maior AB e a maior FR foi observada para a espécie Maytenus robusta.

O corredor de vegetação correspondeu uma verdadeira transição do ambiente ciliar para os inselbergs, possuindo o maior número de indivíduos por área e uma maior heterogeneidade. Pela curva espécie $X$ área (Figura 2) é possível observar a maior heterogeneidade do ambiente corredor de vegetação sobre os demais ambientes. Caracterizando este ambiente, a espécie Callisthene major possui maior valor em área basal. Com relação ao número de indivíduos por área (DA) Faramea nigrescens foi a espécie com maior representatividade e Copaifera langsdorffii obteve maior valor de frequência relativa.

A percentagem de indivíduos arbóreos perfilhados nos distintos ambientes apresentou diferentes resultados, sendo que a maior ocorrência se deu no Inselberg com $23 \%$, seguido de Mata Ciliar com 15\% e Corredor de Vegetação com $14 \%$. O perfilhamento é uma característica natural importante para a ocupação horizontal de algumas populações podendo variar de acordo com o ambiente em que se encontra (SAMPAIO et al., 1998; FIGUEIREDO et al., 2010). Nesse estudo as espécies, Cordiera concolor, Eugenia acutata, Maytenus robusta, Metrodorea stipularis e Myrciaria floribunda, apresentaram indivíduos perfilhados nos três ambientes, no entanto a maior quantidade é encontrada no inselberg. De acordo com Figueiredo et al. (2010) nas florestas tropicais a limitação do crescimento e desenvolvimento das plantas ocorre mais devido às condições não favoráveis do ambiente, do que à limitações fisiológicas.

Cerne, Lavras, v. 20, n. 1, p. 97-104, jan./mar. 2014 
Tabela 1- Parâmetros fitossociológicos das 10 espécies arbóreas de maior VC, amostradas no levantamento em Coqueiral, MG. NI- Número de indivíduos, DA-Densidade absoluta, FA-Frequência absoluta, DoA-Dominância absoluta, VC- Valor de cobertura.

Table 1 - Phytosociological parameters of 10 tree species greater VC sampled in the survey in Coqueiral, MG. NI-Number of individuals, DA-Density absolute FA-Frequency absolute, DoA-Dominance absolute, VC-value coverage.

\begin{tabular}{|c|c|c|c|c|c|c|c|c|c|c|c|c|c|c|c|}
\hline \multirow{2}{*}{ Família/ Espécie } & \multicolumn{5}{|c|}{ Mata Ciliar } & \multicolumn{5}{|c|}{ Inselberg } & \multicolumn{5}{|c|}{ Corredores de Vegetação } \\
\hline & $\mathrm{Ni}$ & $\mathrm{DA}$ & FA & DoA & VC & $\mathrm{Ni}$ & $\mathrm{DA}$ & FA & DoA & VC & $\mathrm{Ni}$ & $\mathrm{DA}$ & FA & DoA & VC \\
\hline \multicolumn{16}{|l|}{ ANACARDIACEAE } \\
\hline Tapirira obtusa (Benth.) J.D.Mitch. & - & - & - & - & - & - & - & - & - & - & 26 & 72,2 & 77,8 & 2,6 & 13,1 \\
\hline \multicolumn{16}{|l|}{ ANNONACEAE } \\
\hline Rollinia sylvatica (A.St.-Hil.) Mart. & - & - & - & - & - & 1 & 5,0 & 20,0 & 2,0 & 9,4 & - & - & - & - & - \\
\hline \multicolumn{16}{|l|}{ COMBRETACEAE } \\
\hline Terminalia glabrescens Mart. & - & - & - & - & - & - & - & - & - & - & 12 & 33,3 & 66,7 & 0,9 & 4,9 \\
\hline \multicolumn{16}{|l|}{ EUPHORBIACEAE } \\
\hline Croton floribundus Spreng. & 28 & 116,7 & 66,7 & 3,0 & 20,3 & - & - & - & - & - & - & - & - & - & - \\
\hline \multicolumn{16}{|l|}{ FABACEAE } \\
\hline Copaifera langsdorffii Desf. & 7 & 29,2 & 66,7 & 1,8 & 8,7 & 30 & 150,0 & 80,0 & 2,9 & 21,4 & 38 & 105,6 & 100,0 & 2,3 & 14,0 \\
\hline Inga vera Willd. & 12 & 50,0 & 50,0 & 1,4 & 9,2 & - & - & - & - & - & - & - & - & - & - \\
\hline Machaerium nyctitans (Vell.) Benth. & 8 & 33,3 & 33,3 & 0,8 & 5,5 & - & - & - & - & - & - & - & - & - & - \\
\hline Platycyamus regnellii Benth. & 10 & 41,7 & 50,0 & 6,3 & 25,5 & - & - & - & - & - & - & - & - & - & - \\
\hline $\begin{array}{l}\text { Tachigali rugosa (Mart. ex Benth.) Zarucchi } \\
\text { \& Pipoly }\end{array}$ & - & - & - & - & - & - & - & - & - & - & 6 & 16,7 & 33,3 & 1,2 & 5,2 \\
\hline \multicolumn{16}{|l|}{ LECYTHIDACEAE } \\
\hline Cariniana legalis (Mart.) Kuntze & 3 & 12,5 & 50,0 & 3,0 & 11,5 & - & - & - & - & - & - & - & - & - & - \\
\hline \multicolumn{16}{|l|}{ MALPIGHIACEAE } \\
\hline Byrsonima laxiflora Griseb. & - & - & - & - & - & - & - & - & - & - & 13 & 36,1 & 77,8 & 1,1 & 5,9 \\
\hline MALVACEAE & - & - & - & - & - & - & - & - & - & - & - & - & - & - & - \\
\hline Luehea divaricata Mart. & - & - & - & - & - & 13 & 65,0 & 80,0 & 0,9 & 7,5 & - & - & - & - & - \\
\hline \multicolumn{16}{|l|}{ MYRSINACEAE } \\
\hline Myrsine umbellata Mart. & - & - & - & - & - & - & - & - & - & - & 21 & 58,3 & 66,7 & 0,6 & 5,5 \\
\hline \multicolumn{16}{|l|}{ MYRTACEAE } \\
\hline Eugenia acutata Miq. & 8 & 33,3 & 33,3 & 1,2 & 6,9 & - & - & - & - & - & - & - & - & - & - \\
\hline Eugenia florida DC. & - & - & - & - & - & 6 & 30,0 & 40,0 & 1,4 & 8,2 & - & - & - & - & - \\
\hline Myrcia tomentosa (Aubl.) DC. & - & - & - & - & - & 5 & 25,0 & 60,0 & 1,4 & 7,7 & - & - & - & - & - \\
\hline $\begin{array}{l}\text { Myrciaria floribunda (H.West ex Willd.) } \\
\text { O.Berg }\end{array}$ & - & - & - & - & - & 29 & 145,0 & 100,0 & 0,2 & 8,6 & - & - & - & - & - \\
\hline Siphoneugena densiflora O.Berg & - & - & - & - & - & - & - & - & - & - & 15 & 41,7 & 77,8 & 0,7 & 4,8 \\
\hline \multicolumn{16}{|l|}{ RUBIACEAE } \\
\hline $\begin{array}{l}\text { Bathysa australis (A.St.-Hil.) Benth. \& } \\
\text { Hook.f. }\end{array}$ & 23 & 95,8 & 50,0 & 1,1 & 11,9 & - & - & - & - & - & - & - & - & - & - \\
\hline Cordiera concolor (Cham.) Kuntze & - & - & - & - & - & 20 & 100,0 & 60,0 & 0,4 & 7,0 & - & - & - & - & - \\
\hline Faramea nigrescens Mart. & - & - & - & - & - & - & - & - & - & - & 43 & 119,4 & 88,9 & 0,7 & 9,6 \\
\hline \multicolumn{16}{|l|}{ RUTACEAE } \\
\hline Metrodorea stipularis Mart. & 9 & 37,5 & 83,3 & 0,7 & 5,5 & 13 & 65,0 & 40,0 & 0,6 & 6,4 & - & - & - & - & - \\
\hline Zanthoxylum rhoifolium Lam. & - & - & - & - & - & 11 & 55,0 & 80,0 & 3,1 & 17,1 & - & - & - & - & - \\
\hline \multicolumn{16}{|l|}{ SALICACEAE } \\
\hline Casearia arborea (Rich.) Urb. & - & - & - & - & - & - & - & - & - & - & 29 & 80,6 & 77,8 & 0,6 & 7,0 \\
\hline \multicolumn{16}{|l|}{ SAPINDACEAE } \\
\hline Maytenus robusta Reissek & - & - & - & - & - & 29 & 145,0 & 100,0 & 0,1 & 8,1 & - & - & - & - & - \\
\hline \multicolumn{16}{|l|}{ URTICACEAE } \\
\hline Cecropia pachystachya Trécul & 10 & 41,7 & 16,7 & 0,8 & 6,3 & - & - & - & - & - & - & - & - & - & - \\
\hline \multicolumn{16}{|l|}{ VOCHYSIACEAE } \\
\hline Callisthene major Mart. & - & - & - & - & - & - & - & - & - & - & 21 & 58,3 & 88,9 & 5,1 & 20,6 \\
\hline
\end{tabular}

Cerne, Lavras, v. 20, n. 1, p. 97-104, jan./mar. 2014 


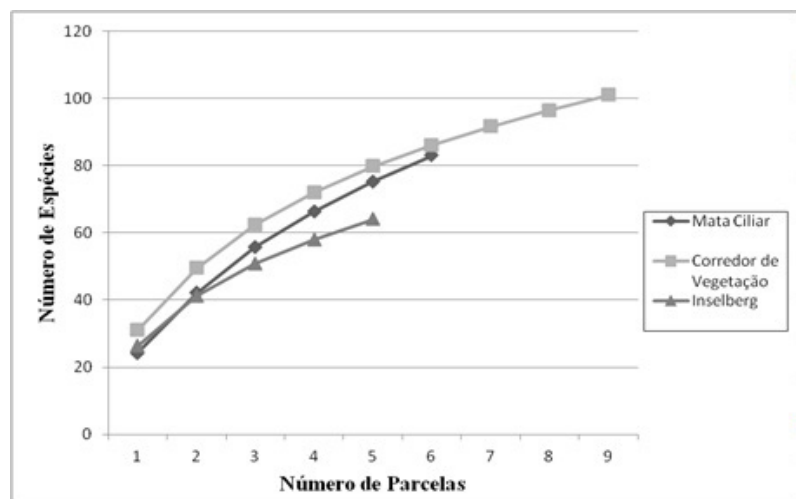

Figura 2- Curva de incremento do número de espécie por parcelas amostradas para aos três ambientes da área de estudo em Coqueiral, MG.

Figure 2 - Curve increase in the number of species per plot sampled for the three environments of the area in Coqueiral, MG.

A amostragem total apresentou um índice de Shannon de 4,43 nats/individuo e um índice de equabilidade de Pielou de 0,86 . Em cada ambiente foi possível encontrar diferentes índices de Shannon: inselbergs 3,61 nats/ individuo; mata ciliar 3,90 nats/individuo; corredor de vegetação 4,02 nats/individuo. Os índices de equabilidade de Pielou dos ambientes foram: mata ciliar 0,76, corredor de vegetação 0,88 e no inselberg 0,86 .
A riqueza e diversidade de espécies podem estar relacionadas com os diferentes mesohabitats abrangido pela amostragem, proporcionado pela topografia, variação das características do solo (umidade, matéria orgânica, nutrientes) e presença de curso d'água. O menor valor para índice de Shannon foi encontrado no inselberg. As características de um ambiente de afloramento rochoso são consideradas das mais estressantes, isso porque suas superfícies rochosas são uma barreira ecológica para a retenção de água e sementes. Pode haver escassez de nutrientes em seus solos, além de sofrerem um forte processo de lixiviação e maior exposição a ventos e insolação em relação a áreas vizinhas (POREMBSKI, 2007; RIBEIRO et al., 2007; CAMPOS et al., 2011; MEDINA et al., 2006).

A análise de espécies indicadoras evidenciou a ocorrência de um padrão de distribuição das espécies em cada ambiente (Tabela 2). Segundo Kent e Coker (1992), a análise de espécies indicadoras parte do pressuposto que para um grupo de amostras haverá um grupo correspondente de espécies que caracterizam aquele ambiente. Os valores de indicação (VI) são baseados na frequência com que a espécie ocorre dentro do grupo e entre os grupos (MCCUNE; MEFFORD, 1999).

De acordo com o resultado das espécies indicadoras, nos inselbergs, estão presentes espécies típicas de ambientes adversos como: Maytenus robusta, considerada uma planta seletiva higrófita, que possui economia hídrica favorável; Luehea divaricata é heliófita, seletiva xerófita e semidecídua;

Tabela 2- Relação das espécies e os valores de indicação (VI) em três grupos de ambientes em um fragmento em Coqueiral, MG. Foram relacionadas somente as espécies que apresentaram valores significativos, segundo teste de "Monte Carlo" para p = 0,05.

Table 2 - List of species and the indication value (VI) in three groups of environments in a fragment Coqueiral MG. Were related only species that showed significant values, the second test of the "Monte Carlo" for $p=0.05$

\begin{tabular}{|c|c|c|c|c|c|c|c|c|}
\hline Mata Ciliar & VI & $\mathrm{p}$ & Corredor de Vegetação & VI & $\mathrm{p}$ & Inselberg & VI & $\mathrm{p}$ \\
\hline Bauhinia longifolia & 67 & 0,005 & Siphoneugena densiflora & 69 & 0,004 & Maytenus robusta & 89 & 0,001 \\
\hline Croton floribundus & 62 & 0,024 & Amaioua intermedia & 67 & 0,008 & Myrciaria floribunda & 81 & 0,003 \\
\hline Senna macranthera & 50 & 0,028 & Protium spruceanum & 67 & 0,010 & Luehea divaricata & 80 & 0,001 \\
\hline Machaerium hirtum & 50 & 0,026 & Callisthene major & 66 & 0,011 & Eugenia cerasiflora & 77 & 0,003 \\
\hline Inga vera & 50 & 0,022 & Faramea nigrescens & 63 & 0,021 & Zanthoxylum rhoifolium & 73 & 0,003 \\
\hline Guapira hirsuta & 50 & 0,026 & Casearia arbórea & 62 & 0,017 & Luehea grandiflora & 49 & 0,040 \\
\hline Celtis brasiliensis & 50 & 0,028 & Myrsine umbellata & 62 & 0,012 & Zanthoxylum fagara & 47 & 0,030 \\
\hline Cariniana legalis & 50 & 0,026 & Tapirira obtusa & 60 & 0,048 & & & \\
\hline \multirow[t]{2}{*}{ Cabralea canjerana } & 50 & 0,023 & Daphnopsis utilis & 56 & 0,030 & & & \\
\hline & & & Byrsonima laxiflora & 50 & 0,050 & & & \\
\hline
\end{tabular}

Cerne, Lavras, v. 20, n. 1, p. 97-104, jan./mar. 2014 
Luehea grandiflora, seletiva higrófita, apresenta dispersão irregular e descontínua, frequente em solos rochosos; Zanthoxylum rhoifolium, semidecídua, seletiva higrófita e heliófita, ocorrem preferencialmente em terrenos íngremes e de rápida drenagem (FERNANDES, 2000). No inselberg formam-se centros de diversidades de plantas que possuem certos tipos funcionais, como plantas tolerantes a dessecação, que estão bem adaptados para a sobrevivência em ambientes hostis (POREMBSKI, 2007; POREMBSKI; BARTHLOTT, 2000).

O diagrama de ordenação mostra a distribuição das parcelas (Figura 3) e das variáveis ambientais nos dois primeiros eixos de ordenação. Os autovalores para os dois primeiros eixos foram de 0,471 e 0,379 , respectivamente, indicando que existe substituição de espécies no gradiente de acordo com a variação ambiental. A variância acumulada nos dois primeiros eixos de ordenação foi de $18 \%$. Este valor indica que a ordenação deixou uma considerável variância remanescente sem explicação. No entanto segundo ter Braak (1988), esse resultado é normal em dados de vegetação e não compromete as análises das relações espécies-ambiente. Foi possível observar também que no diagrama houve uma separação entre os três ambientes, caracterizando uma alta substituição de espécies. As correlações das variáveis ambientais com o primeiro eixo foram: $\mathrm{Al}(0,929), \mathrm{SB}$ $(-0,907), \mathrm{Ca}(-0,900), \mathrm{pH}(-0,745)$ e $\mathrm{MO}(0,677)$.

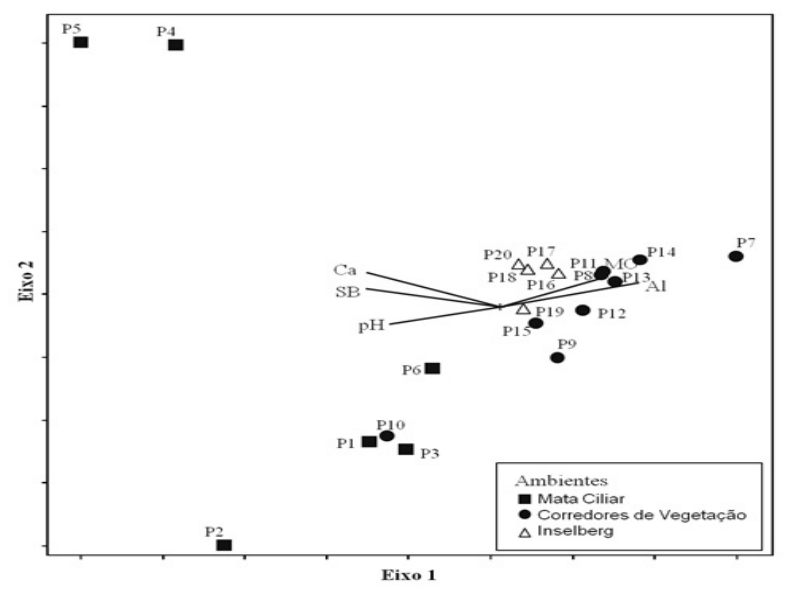

Figura 3- Diagrama de ordenação produzido pela análise de correspondência canônica (CCA) da abundância de 170 espécies distribuídas em 20 parcelas em Coqueiral, MG, e suas relações com variáveis ambientais.

Figure 3- Ordination diagram produced by canonical correspondence analysis (CCA) of abundance of 170 species in 20 plots in Coqueiral, $M G$, and their relationships with environmental variables

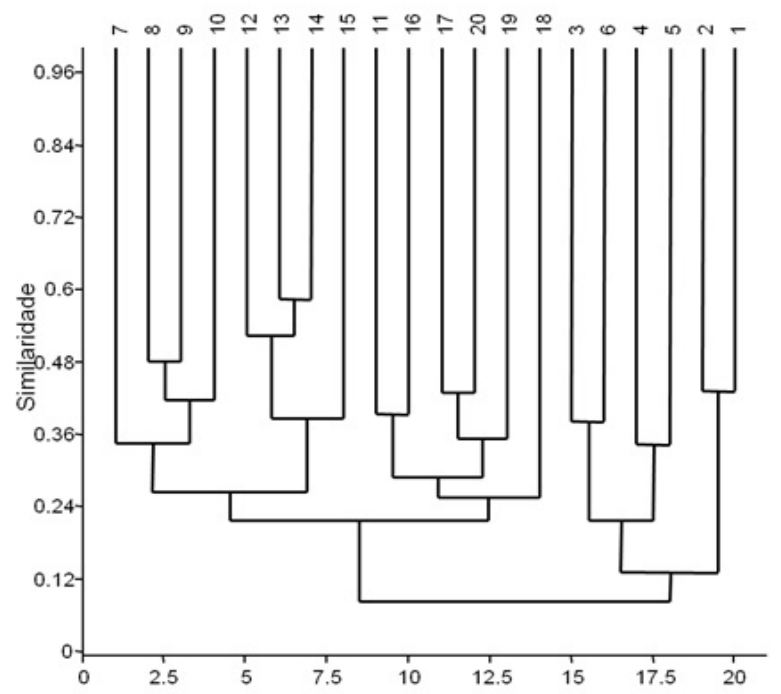

Figura 4- Dendorgrama de similaridade (Bray-Curtis) baseado na abundância das espécies distribuídas nas 20 parcelas da área de estudo em Coqueiral, MG.

Figure 4- Dendorgrama similarity (Bray-Curtis) based on the abundance of species distributed in 20 plots of the study area in Coqueiral, $M G$.

A diferenciação entre os ambientes pode ser confirmada através do dendrograma de similaridade (Figura 4). Observa-se que as parcelas alocadas na mata ciliar apresentam menor similaridade com as parcelas do inselberg e corredor de vegetação.

Embora a parcela 11 pertença ao ambiente de corredor de vegetação, esta possui maior similaridade com as parcelas do inselberg. Essa similaridade está associada ao fato da proximidade geográfica, sendo uma parcela de transição entre corredor de vegetação e inselberg.

As características edáficas e a disponibilidade hídrica de cada ambiente foram decisivas para a composição e predominância das espécies nos distintos ambientes. Critérios de solo, como drenagem, profundidade de solo, presença de afloramentos rochosos e disponibilidade de nutrientes são importantes para a classificação de fitofisionomias (OLIVEIRA-FILHO; FLUMINHAN-FILHO, 1999; POREMBSKI, 2007).

A heterogeneidade, qualidade de habitat e complexidade estrutural são as principais características do ambiente que influenciam na distribuição das espécies e nos padrões de organização biológica. Assim a classificação

Cerne, Lavras, v. 20, n. 1, p. 97-104, jan./mar. 2014 
da vegetação em um fragmento dependerá não somente da composição de espécies, mas também das variáveis ambientais que influenciam sobres estas.

$\mathrm{O}$ fragmento estudado em Coqueiral-MG apresentou considerável heterogeneidade ambiental, condicionada principalmente pelos atributos do solo, e evidenciada pela análise de correspondência canônica e pelo dendrograma. Esta heterogeneidade ambiental é refletida na diversidade e abundância das espécies arbóreas, diferenciando os ambientes e caracterizando essa área de inselberg como um ecótono.

\section{REFERÊNCIAS}

BURKE, A. Island-matrix relationships in Nama Karoo inselbergs landscapes. Part I: Do inselbergs provide a refuge for matrix species? Plant Ecology, Dordrecht, v.160, n.12, p.79-90

CAMPOS, M.C.R.; TAMASHIRO, J.Y.; ASSIS, M.A.; JOLY, C.A. Florística e fitossociologia do componente arbóreo da transição Floresta Ombrófila Densa das Terras Baixas - Floresta Ombrófila Densa Submontana do Núcleo Picinguaba/PESM, Ubatuba, sudeste do Brasil. Biota Neotropica, São Paulo, v.11, n.2, p.301312, 2011.

EMBRAPA- Empresa Brasileira de Pesquisa Agropecuária. Centro Nacional de Pesquisa de Solos. Manual de métodos análise de solos. Rio de Janeiro, 2002. 400p.

FELFILI, J.M.; NASCIMENTO, A.R.T.; FAGG, C.W.; MEIRELLES, E.M. Floristic composition and community structure of a seasonally deciduous forest on limestone outcrops in Central Brazil. Revista Brasileira de Botânica, São Paulo, v.30, n.4, p.611-621, out./dez. 2007.

FERNANDES, A. Fitogeografia brasileira. Fortaleza, 2a edição. 2000. 340p.

FIGUEIREDO, L.S.; FERRAZ, E.M.N.; RODAL, M.J.N.; PIMENTEL, R.M.M.; ARAÚJO, E. Sítios de estabelecimentos e relações alométricas em populações lenhosas da caatinga. Revista de Geografia, Recife, v.27, n.2, p.155-167. 2010.

GOMES, P.; ALVES, M. Floristic diversity of two crystalline rocky outcrops in the Brazilian northeast semi-arid region. Revista Brasileira de Botânica, São Paulo, v. 33, n.4, p.661-676. 2010.
HAMMER, Ø. 2009. PAST: Paleontological Statistics Software Package for Education and Data Analysis. Palaeontologia Electronica.

JOLY, A.B. Conheça a vegetação brasileira. São Paulo. EDUSP. 1970. 181p.

KENT, M.; COKER, P. Vegetation description and analysis: a practical approach. British Library Cataloguing, London. 1992. 363p.

LARSON, D.W., MATTHES, U.; KELLY, P.E. Cliff ecology: pattern and process in cliff ecosystems. Cambridge Studies in Ecology. Cambridge University Press, Cambridge. 2000. 335p.

LOBO, P.C.; JOLY, C.A. Aspectos ecofisiológicos da vegetação de mata ciliar do sudeste do Brasil. In:RODRIGUES, R.R.; LEITÃO FILHO, H.F. (Eds.) Matas Ciliares: conservação e recuperação. São Paulo: EDUSP, FAPESP, 2000. p.143-157.

McCUNE, B.; MEFFORD, M.J. Multivariate analysis of ecological data. Gleneden Beach, MjM Software. 1999. 237p.

MEDINA, B.M.O.; RIBEIRO, K.T.; SCARANO, F.R. Plant-Plant and Plant-Topography Interactions on a Rock Outcrop at High Altitude in Southeastern Brazil. Biotropica, Whasington, v.38, n.1, p. 27-34, 2006.

MUELLER-DOMBOIS, D.; ELLENBERG, H. Aims and Methods of Vegetation Ecology. New York, John Wiley \& Sons. 1974. 547p.

OLIVEIRA-FILHO, A.T.; FLUMINHAN-FILHO, M. Ecologia da Vegetação do Parque Florestal Quedas do Rio Bonito. Cerne, Lavras, v.5, n.2, p.51-64, 1999.

OLIVEIRA FILHO, A.T.; FONTES, M.A.L. Patterns of floristic differentiation among Atlantic forests in southeastern Brazil, and the influence of climate. Biotropica, Washington, v.31, p. 783-810. 2000.

OLIVEIRA FILHO, A. T.; RATTER, J. A. Padrões florísticos das matas ciliares da região do Cerrado e a evolução das paisagens do Brasil Central durante o Quaternário Tardio. In: Rodrigues, R.R. \& Leitão Filho, H.F. (eds.). Matas ciliares: conservação e recuperação. EDUSP, São Paulo, 2000. p. 73-89.

Cerne, Lavras, v. 20, n. 1, p. 97-104, jan./mar. 2014 
PARMENTIER, I. Ecology and Distribution of Melastomataceae in African Rain Forest Inselbergs.

Biotropica, Washington, v.37, n.3, p.364-372, 2005.

PARMENTIER, I.; HARDY, O.J. The impact of ecological differentiation and dispersal limitation on species turnover and phylogenetic structure of inselberg's plant communities. Ecography, Copenhagen v. 32, p.613-622, 2009.

PARMENTIER, I.; STÉRVART, T.; HARDY, O.J. The inselberg flora of Atlantic Central Africa. I. Determinants of species assemblages. Journal of Biogeography, Oxford v.32, p. 685-696, 2005.

PINHEIRO, M.H.O.; MONTEIRO, R. Análise estrutural e considerações sobre a dinâmica sucessional de dois fragmentos florestais semideciduais do Jardim Botânico Municipal de Bauru, SP, Brasil. Acta Botânica

Brasílica, Feira de Santana, v.23, n.4, p.968-975. 2009.

POREMBSKI, S. Tropical inselbergs: habitat types, adaptive strategies and diversity patterns. Revista Brasileira de Botânica, São Paulo, v.30, n.4, p. 579586, 2007.

POREMBSKI, S.; BARTHLOTT, W. Granitic and gneissic outcrops (inselbergs) as centers of diversity for desiccation-tolerant vascular plants. Plant Ecology, Boston, v.151, p.19-28, 2000.

RIBEIRO, K.T.; MEDINA, B.M.O.; SCARANO, F.R. Species composition and biogeographic relations of the rock outcrop flora on the high plateau of Itatiaia, SE-

Brazil. Revista Brasileira de Botânica, São Paulo, v.30, n.4, p. 623-639, 2007.

SAMPAIO, E.V.S.B.; ARAÚJO, E.L.; SALCEDO, J.H.; TIESEN, H. Regeneração da vegetação de caatinga após corte e queima, em Serra Talhada-PE. Pesquisa Agropecuária Brasileira, São Paulo, v. 33, n. 5, p. 621632. 1998.

SOUZA, J.S.; ESPÍRITO-SANTO, F.D.B.; FONTES, M.A.L.; OLIVEIRA FILHO, A.T.; BOTEZELLI, L. Análise das variações florísticas e estruturais da comunidade arbórea de um fragmento de floresta semidecídua às margens do rio Capivarí, Lavras-MG.

Cerne, Lavras, v. 20, n. 1, p. 97-104, jan./mar. 2014
Revista Árvore, Viçosa, v.27, p.185-206. 2003.

TER BRAAK, C.J.F. The analysis of vegetation environment relationships by canonical correspondence analysis. Vegetatio, Dordrecht, v. 69, n. 3, p. 69-77, 1988.

VILELA, E.A.; RAMALHO, M.A.P. Análise das temperaturas e precipitações pluviométricas de Lavras MG. Ciência e Prática, Bebedouro, v.3, p.71-79. 1979.

Recebido: 04 de junho de 2012; aceito: 12 de agosto de 2013. 